\title{
Long Irregular LDPC Coded OFDM with Soft Decision
}

\author{
Madhusmita Mishra \\ Department of Electronics and \\ Communication Engineering \\ National Institute of Technology \\ Rourkela-769008, India
}

\author{
Sarat Kumar Patra \\ Department of Electronics and \\ Communication Engineering \\ National Institute of Technology \\ Rourkela-769008, India
}

\author{
Ashok Kumar Turuk \\ Department of Computer \\ Science and Engineering \\ National Institute of Technology \\ Rourkela-769008, India
}

\begin{abstract}
OFDM with quadrature amplitude modulation (QAM) technique can be used for high speed optical applications. Increasing the order of modulation, the bit error rate (BER) increases. Forward Error correction (FEC) coding like LDPC coding is generally used to improve the BER performance. LDPC provides large minimum distance and also the power efficiency of the LDPC code increases significantly with the code length. In this paper we have compared the Soft decision and Hard decision algorithms of LDPC codes. A long Irregular LDPC code is simulated over the BIAWGN channel demonstrating the fact that LDPC coded OFDM with soft decision decoding provides very lower bit error rate as well as a larger gain in transmitter power and thus making the link more power efficient than the case with hard decision decoding. Through simulation, we have shown the advantages of using this long Irregular LDPC coded OFDM link in Optical wireless communication (OWC).
\end{abstract}

\section{General Terms}

Orthogonal Frequency Division Multiplexing, Low Density Parity check coding, Quadrature Amplitude Modulation.

\section{Keywords}

Long Irregular LDPC code, BIAWGN channel, Soft decision decoding, Message-passing (MP) algorithm, Error rate floors.

\section{INTRODUCTION}

OFDM provides an effective and low complexity means for eliminating inter-symbol interference for transmission over frequency selective fading channels. In OFDM the subcarrier frequencies are chosen in such a way that the signals are mathematically orthogonal over one OFDM symbol period [2]. During the past decades, channel coding has been used extensively in most digital transmission systems, from those requiring only error detection, to those needing very high coding gains. In the past, optical communication systems have ignored channel coding, until it became clear that it could be a powerful, yet inexpensive, tool to add margins against line impairments such as amplified spontaneous emission (ASE) noise, channel cross talk, nonlinear pulse distortion, and fiber aging-induced losses[3,7]. Nowadays, channel coding is a standard practice in many optical communication links.

For any channel, the bandwidth, data rate, noise and error rate are related to each other. Greater the bandwidth, greater will be the cost. All transmission channels of any practical interest are limited in bandwidth due to the constrained physical properties of the transmission medium. Inorder to use bandwidth efficiently, in a digital transmission, it is required to obtain higher possible data rate at a particular limit of error rate. But, noise is the main constraint in obtaining higher possible data rate at a given error rate. If binary signals are transmitted then the supported data rate will be twice the bandwidth. But, using multilevel signaling the data rate can be increased by a factor of $\log _{2} \mathrm{M}$, where $\mathrm{M}$ is the no of signal levels. Here we have used QAM modulated OFDM to achieve high data rate.

As data rate increases, the duration of bits gets shorter as a result of which more bits are affected by a given pattern of noise concluding the statement that higher data rate leads to higher error rate. The solution now is to increase the signal to noise ratio (SNR), which sets the upper bound on the achievable data rate. Shannon's formula assumes only white noise (thermal noise) and it does not account for the impulse noise or distortion due to attenuation and delay. While the Shannon's formula represents the theoretical maximum that can be achieved, but in practice much lower rates are achieved. Similarly it does not suggest rather provide a yardstick for finding a suitable signal code to achieve error free transmission [12]. In this paper we have used long Irregular LDPC code which answers all the questions aroused from Shannon's theorem, while applied to several practical communication applications.

Rest of the paper is organized as follows: Section 2 describes the Problem formulations. An overview of LDPC codes is given in Section 3. Comparison of performance of LDPC coded OFDM is made in Section 4. Finally few conclusions are drawn in Section 5.

\section{PROBLEM FORMULATIONS}

For a signal transmitted without coding, an SNR of around 10.5 to $11 \mathrm{~dB}$ is required to achieve a BER of $10^{-6}$. Use of Reed Solomon (RS) code was resulting in $4 \mathrm{~dB}$ of coding gain and leaving of $6 \mathrm{~dB}$ gain was still there. Even $\mathrm{BCH}$ and convolutional codes were going down to $5 \mathrm{~dB}$ and were not able to go down to $1 \mathrm{~dB}$ or $2 \mathrm{~dB}$. So, for a long time there is a belief that it is not possible to do any better than this $4 \mathrm{~dB}$ or $5 \mathrm{~dB}$ of coding gain. Recently Turbo and LDPC codes are discovered to achieve large coding gains. A comparative study of existing coding techniques is presented in Table 1.

Though Turbo and LDPC codes belong to the family of compound codes $[7,8]$, the LDPC codes have the following advantages over Turbo codes. Firstly, in Turbo code due to the presence of low weight code words there is a chance of mistaken of a codeword for its nearby codeword due to noise in channel. But, the construction of LDPC code avoids the presence of low weight codewords.

Here we have used a very long irregular code which has excellently used the distance properties of LDPC codes. The presence of low weight codewords causes the error floor region of Turbo codes to be around a bit error rate (BER) of $10^{-5}$ to $10^{-6}$. While in contrast the LDPC codes have error floor region around $10^{-6}$ to $10^{-8}$. 
Secondly, the use of BCJR algorithm aids the computational complexity of Turbo codes since no of computations in each recursion of the BCJR algorithm scales linearly with the no of states in the trellis. Commonly used Turbo codes have 16 states in the trellis, where as the LDPC decoders use a simple parity check trellis having only 2 states. Also the extreme sparseness of the parity check matrix results in a low complexity LDPC decoding algorithm.

Thirdly, being parallelizable, the speed of decoding of LDPC code is greater than that of Turbo code. Fourthly, the LDPC decoder declares a decoding failure, whenever unable to correctly decode, where as the Turbo decoders perform extra computations to declare a stopping criterion, which depends upon the establishment of a threshold. Finally LDPC code of almost any rate and block length can be designed only from the specification of target parity check matrix, while the turbo code rate is depending on a puncturing schedule.

In the LDPC codes, parity check matrix is generated randomly and it answers these two questions raised by Shannon's paper [15]. The first one is -"How the codes can be constructed which will satisfy the upper bound of Channel coding theorem by Shannon" and the second one is- "How efficiently the maximum likelihood decoding can be done for the code"?

The answer to the first question is random construction of code, which the LDPC codes have and the answer to the second question is use of a code with large block length. Here, this is the motivation behind using a long Irregular LDPC code for making the link power efficient by achieving larger coding gain.

\section{OVERVIEW OF LDPC CODES}

Low-density parity-check (LDPC) codes are linear block codes specified by a parity check matrix $\mathrm{H}$ containing mostly 0 's and only a small number of 1 's.

A regular $\left(N, w_{c}, w_{r}\right)$ LDPC code [5,14] is a code of block length $N$ with a $M_{p} \times N$ parity check matrix where each column contains a small fixed number, $w_{c} \geq 3$ of 1 's and each row contains a small fixed number, $w_{r} \geq w_{c}$ of 1's.

Low-density implies that $w_{c} \ll M_{p}$ and $w_{r} \ll N$. Number of ones in the regular parity check matrix is given by

$$
w_{c} \cdot N=w_{r} \cdot M_{p}
$$

For an irregular low-density parity-check code [6] the degrees of each set of nodes are chosen according to some distribution. In the construction of irregular LDPC code, the First step involves selecting a profile that describes the desired number of columns of each weight and the desired number of rows of each weight. Second step includes a Construction method, i.e. algorithm for putting edges between the vertices in a way that satisfies the constraints. The edges are placed "completely at random" subject to the profile constraints. The no of 1's in the Irregular LDPC code matrix is given by

$$
M_{p}\left(\sum_{i} w_{i} i\right)=N\left(\sum_{i} u_{i} i\right)
$$

Where $M_{p}$ is the no of parity check constraints, $N$ is the length of the code, $u_{i}$ and $w_{i}$ are the column and row degree distributions respectively. If $\mathbf{H}$ and $\mathbf{C}$ are the parity check matrix and the codeword respectively, then the codeword constraint is given by

$$
\left[H C^{T}\right]=[0]
$$

Where each row of $H$ corresponds to a parity check equation and each column of $H$ corresponds to a bit in the codeword. The $(j, i)^{\text {th }}$ entry of $\mathrm{H}$ is 1 , if the ith codeword bit is included in the jth parity check equation. More than one parity check matrices may satisfy the above constraint and hence there may be more than one parity check matrices describing a particular code. But, any possible two parity check matrices representing the same code may not have the same no of rows. In an $H$ matrix, if there is no mathematical relationship between rows, it will be said to have linearly independent rows and otherwise to have dependent rows. More the parity check constraints, lesser is the number of satisfied codewords provided the parity check equations are linearly independent.

The LDPC codes can be differentiated from classical block codes [9 10] by the sparseness of their parity check matrix. This property of $H$ is essential for an iterative decoding complexity that increases only linearly with the code length. The classical block codes can also work with iterative algorithms, but finding a sparse $H$ matrix for existing codes of them is impractical.

In case of LDPC codes, first a parity check matrix with required properties is designed and then it is encoded with suitable existing encoding algorithms and then decoded iteratively using a tanner graph representation of their parity check matrix using any one of the existing iterative algorithms.

Classical block codes are decoded with ML (Maximum Likelihood) decoding algorithm. Reviewing the use of decoding techniques, one can state that if no extra information is known about the codeword $\mathbf{C}$ other than the information from channel, the ML decoder will be the best one to choose the correct codeword successfully. But, if the decoder has a priori information about $\mathrm{C}$ then it is desirable to use a more sophisticated decoding like Maximum a posteriori (MAP) or block-MAP decoder. For these decoders, the value of $\mathrm{K}$ (no of message bits) does not have to be very large, so as to make decoding completely impossible, since large block lengths results in large parity check and generator matrices. 
Instead the iterative decoding algorithms used in case of LDPC codes decoding can handle large block lengths and produce accurate estimates of the Probability $\left(C_{i} /\right.$ channel output for $C_{i}$ ) using repeated low-complexity processes. The Irregular feature of the parity check matrix of LDPC codes enable them to show high performance with respect to Shannon limit [11].

\subsection{Encoding of LDPC codes}

The set of valid codewords are those which satisfies the parity check constraints. The generator matrix is used to map the messages to the codewords $[11,13,14]$.

The $(j, i)^{\text {th }}$ entry of $G$ is ' 1 'if the $j$ th message bit plays a role in determining the $i^{\text {th }}$ codeword. All possible linear combination of the rows of $\mathrm{G}$ gives the set of codewords for the code with generator $G$. The generator matrix $(\mathrm{G})$ satisfies the following equation.

$$
G H^{T}=0
$$

The steps for encoding after designing the parity check matrix are as below:

i. Put $H$ in the row-echelon form to get a new matrix as $H_{g r}$.

ii. Then convert $H_{g r}$ to reduced row- echelon form denoted by $H_{g r r}$.

iii. Then put $H_{g r r}$ into standard form: $H_{g r r}=[A$ $\left.I_{N-K}\right]$, where $A$ is $(N-K)$ by $K$ binary matrix and $I_{N-K} C=\underline{m} G$ is the identity matrix of order $N-K$.

iv. Then the generator matrix will be $G=\left[I_{K} A^{T}\right]$

v. Then the message is mapped to the codeword by the relation $C=\underline{m} G$, where $\underline{m}$ is message vector to be encoded.

\subsection{Decoding of LDPC codes}

To give an introduction to LDPC decoding algorithms, we must say that Gallager [14] has also provided a decoding algorithm that is typically near optimal. The iterative decoding algorithms used in LDPC decoding measures the probability distribution of variables in graph based models and come under different names depending on the circumstances. But, collectively they are termed as messagepassing (MP) algorithms [11,13].

The task of the decoder is to detect and correct the flipped bits during transmission over any channel. Every received word that does not satisfy equation (3) above will not be a codeword and hence the design of $H$ keeps an important pace in reducing the complexity of decoding errors. In message-passing algorithm, messages pass back and forth between the bit and check nodes until the equation (3) is satisfied.
The hard decision message-passing algorithm is known as bitflipping algorithm and the passed messages are binary in nature $[11,13]$. A binary hard decision about each received bit is made by the detector. This is implemented in [1]. The problem with this type of decoding is that the decoder performance is not good. The reason for this is it does not take the likelihood values into account rather it takes a binary decision about the occurrence of a transmitted bit. Hence, the bit error rate (BER) plots have not so good error rate floors.

The sum-product algorithm (SPA) is the soft-decision message-passing algorithm. It is similar to the bit-flipping algorithm except that the passed messages between bit node and check node are probabilities. It has two versions, one is probability domain version that computes a posteriori probability (APP) and another is log-domain version that computes log-likelihood ratios (LLRs). Both of these versions use likelihood values in decoding. But, the log-domain version is better since in log-domain the complex multiplications are converted to additions resulting in low cost implementation of the decoder with a more stable performance $[9,11,13]$.

Our effort here utilizes the log-domain SPA. So our discussion here is limited to log-domain SPA which is explained below.

\subsubsection{Sum-product Algorithm (Soft-decision Algorithm)}

If we are transmitting a Codeword with $\mathrm{N}$ number of bits, then the APP is the probability that the given bit in the transmitted codeword is equal to 1 or 0 , given the channel output for that bit. Then the APP ratio or the likelihood ratio (LR) is given by

$$
l\left(c_{j}\right) @ \frac{\operatorname{Pr}\left(c_{j}=0 / \text { channeloutputfor } 0\right)}{\operatorname{Pr}\left(c_{j}=1 / \text { channeloutputfor } 1\right)}
$$

Then the log-APP ratio or the log-likelihood ratio(LLR) will be given by

$$
L\left(c_{j}\right) @ \log \left(\frac{\operatorname{Pr}\left(c_{j}=0 / \text { channeloutputfor } 0\right)}{\operatorname{Pr}\left(c_{j}=1 / \text { channeloutputfor } 1\right)}\right)
$$

The output of the band pass demapper [1] is $L\left(c_{j}\right)$, where $\left(c_{j}\right)$ is the jth bit of the transmitted codeword C. The three key parameters in this algorithm are $L\left(r_{i j}\right), L\left(q_{j i}\right)$ and $L\left(Q_{j}\right)$. The $L\left(q_{j i}\right)$ is initiated as $L\left(q_{j i}\right)=L\left(c_{j}\right)$ and these three parameters are updated using the following equations for each iteration. 


$$
\begin{aligned}
& L\left(r_{i j}\right)=2 a \tanh \left(\prod_{j^{\prime} \in v_{i} / j} \tanh \left(\frac{1}{2} L\left(q_{j^{\prime} i}\right)\right)\right) \\
& L\left(q_{j i}\right)=L\left(c_{j}\right)+\sum_{i^{\prime} \in c_{j} / i} L\left(r_{i^{\prime} j}\right) \\
& L\left(Q_{j}\right)=L\left(c_{j}\right)+\sum_{i \in c_{j}} L\left(r_{i j}\right)
\end{aligned}
$$

(7)

\section{PERFORMANCE COMPARISION OF LDPC CODED OFDM}

The simulation environment is same as in [1] except that the LDPC decoding type is soft decision type using log domain SPA as discussed in Section 3.

This system is simulated entirely referring to the block diagram in Figure.1 of reference [1] by introducing the LDPC encoder block at label ' $\mathrm{A}$ ' and LDPC decoder block at label ' $Q$ ' and taking AWGN channel. Here the simulation is carried out using Matlab.We consider an irregular LDPC code with parity check matrix of size 32400 by 64800 . Parity-check matrix of the LDPC code is stored as a sparse logical matrix. The system was simulated for OFDM with 8, 16, 32 and64 QAM. Columns 32401 to 64800 are a lower triangular matrix. Only the elements on its main diagonal and the sub diagonal immediately below are 1 's. Since the last $N-K$ columns of the parity check matrix is of lower triangular type, it is referred as the forward substitution method of encoding. This is discussed in section 3 . The code rate is $1 / 2$.

In reference [1], the LDPC decoder is of hard decision type and here the LDPC decoder is of soft decision type .The information is binary in nature. The channel is thus can be referred as a BIAWGN channel. The Fig 1 shows the BER performance of 16 and 32 and 64 QAM modulated LDPC coded OFDM in hard-decision mode [1]. The number of iterations performed at the decoder is 40 . As the number of iterations increases, a more appropriate converged value is obtained. Figure 2 and Fig 3 shows the BER performance with soft decision LDPC decoding for decoder iterations of 10 and 20 respectively. It is observed from the three figures that in the lower SNR region, LDPC coded 16-QAM modulated OFDM signal gives error floor around $10^{-5}$ with 40 decoder iterations of hard decision decoding nearly between $12-14 \mathrm{~dB}$ of SNR, while with soft decision decoding it is around $10^{-7}$ at approximately $8 \mathrm{~dB}$ of SNR with lesser number of decoder iterations of 10 and around $10^{-9}$ nearly at $8 \mathrm{~dB}$ of SNR with decoder iterations of 20 . This clearly illustrates the fact that with soft decision decoding increase in decoder iterations also improves error floor performance. Hence with soft decision decoding there is a gain in power compared to hard decision case with lesser number of decoder iterations.

\section{CONCLUSION}

With soft-decision decoding the BER performance of this long Irregular LDPC code is much better than that with hard decision decoding for all the higher order QAM modulated OFDM signals. The error floor region with this long block length Irregular LDPC code is within $10^{-5}$ to $10^{-6}$ with hard decision decoding and within $10^{-7}$ to $10^{-8}$ with soft decision
Table 1. Comparative study of coding techniques

\begin{tabular}{|l|l|l|l|}
\hline $\begin{array}{l}\text { Coding } \\
\text { Technique }\end{array}$ & $\begin{array}{l}\text { Historical } \\
\text { Timeline }\end{array}$ & $\begin{array}{l}\text { Coding } \\
\text { Gain } \\
\text { (approx) }\end{array}$ & $\begin{array}{l}\text { Distance from } \\
\text { Shannon limit } \\
\text { (approx) }\end{array}$ \\
\hline $\begin{array}{l}\text { Convolutional } \\
\text { Code }\end{array}$ & 1955 & $5 \mathrm{~dB}$ & $5 \mathrm{~dB}$ \\
\hline Regular LDPC & 1960 & $9 \mathrm{~dB}$ & $1 \sim 1.5 \mathrm{~dB}$ \\
\hline Turbo Code & 1993 & $9.3 \mathrm{~dB}$ & $0.7 \mathrm{~dB}$ \\
\hline $\begin{array}{l}\text { Irregular } \\
\text { LDPC }\end{array}$ & 1999 & $10.5 \mathrm{~dB}$ & $0.0045 \mathrm{~dB}$ \\
\hline
\end{tabular}

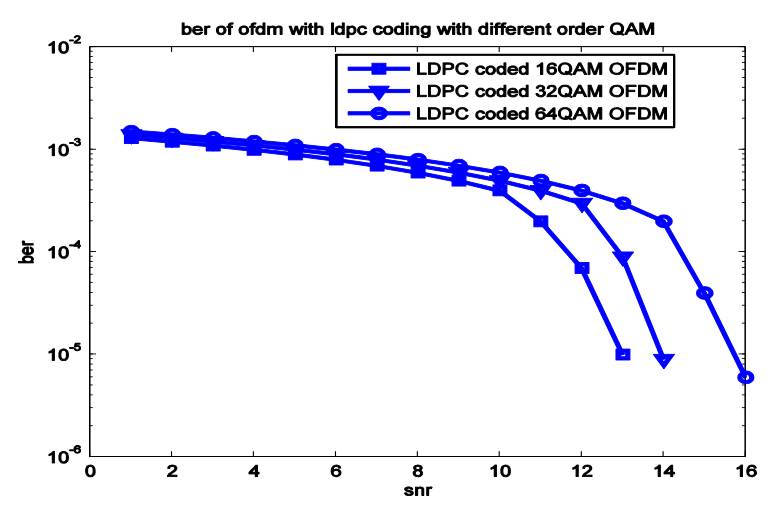

Figure.1: BER of $(32400,64800) L D P C$ coded OFDM for 16, 32 and 64 QAM With Hard-decision Decoding

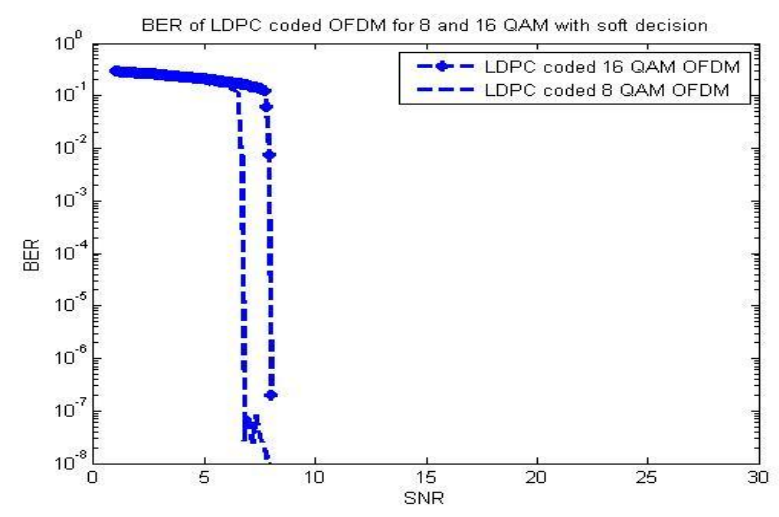

Figure.2: BER of LDPC(32400,64800)coded OFDM for 8 and 16 QAM with Soft Decision Decoding

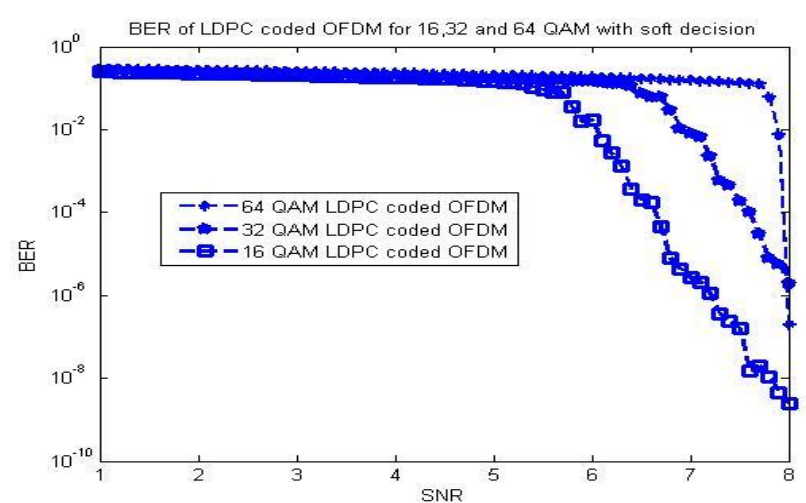

Fig. 3: BER of LDPC (32400, 64800) coded OFDM for 16,32and64 QAM with Soft Decision Decoding 
decoding of 10 decoder iterations and $10^{-5}$ to $10^{-9}$ with soft decision decoding of 20 decoder iterations. This is a better result as far as better coding gain and error floor region is considered. The QAM modulated OFDM signal can be a good match to the higher information carrying capacity of optical carriers. But, the BER increases as the modulation order increases. Using a long Irregular LDPC code can reduce the BER along with approaching the Shannon's limit. Hence, we can use this code with higher order QAM modulated OFDM for improving performance of optical AWGN channel in Free space optical communication . With the advantage of OFDM to deal with Inter Symbol Interference (ISI), this work can also be extended to deal with ISI due to chromatic dispersion and polarization mode dispersion in Optical communication systems. Further due to good error floor region with soft decision it can be used to avoid effect of four wave mixing in BER performance. In another scenario, without taking the weather effects into account, the deep-space channel is a perfect additive white Gaussian noise channel. Also, deepspace communications do not care about the block lengths. Therefore, we can use this long irregular code in deep-space communication applications to get better results, since larger block length results in higher minimum distance between Codewords and thus increasing the error correction capability.

\section{FUTER RESEARCH DIRECTION}

With the above method of encoding, the encoding complexity can become prohibitively complex as we move to long codes of length of the order of $10^{5}$ or $10^{6}$. Use of a structured parity check matrix can be useful to reduce this implementation complexity. This is the current topic of investigation.

\section{REFERENCES}

[1] Mishra, M.; Patra, S.K.; Turuk, A.K. ,'Performance of Power efficient LDPC coded OFDM over AWGN channel", RAIT 2012, Page(s): 185 - 191.

[2] Eldomabiala, Mathias, coinchon, KarimMaouche, "Studyof OFDM Modulation," Ierucom Institute, December, 1999.

[3]. Enrico Forestieri, CommunicationTheoryandTechniques, 2005 .

[4] C. Berrou and A. Glavieux, "Near optimum error correcting coding and decoding: Turbo codes," IEEE Trans. Commun., vol. 44, pp.1261-1271, Oct. 1996

[5] R. Gallager,"Low-density parity-check codes," IEEE Trans. Inform.Theory, vol. 8, no. 1, pp. 21-28, Jan. 1962.

[6] T. J. Richardson and R. L. Urbanke, "Efficient encoding of low-density parity-check codes," IEEE Trans. Inform. Theory, vol. 47, no. 2, pp. 638-656, Feb. 2001.

[7 William Shieh, Ivan Djordjevic,’OFDM for Optical Communication", Academic Press, October, 2009.

[8] Shu Lin and Daniel J.Costello ,"Error Control Coding", Jr.second. edition, Prentice Hall, 2004.

[9] Ferrari and Raheli, "Ldpc coded Modulations", SPRINGER Publication, 2009.

[10] R.E.Blahut, "Algebraic codes for data transmission", 1st edition, Cambridge university press, 2003.
[11] Bernhard M.J.Leiner, "LDPC codes - a brief Tutorial", April 8, 2005.

[12] William Stallings, "Wireless Communication and Networks", Pearson Education,2002.

[13] William E. Ryan, "An Introduction to LDPC codes", August 19, 2003.

[14] Robert G. Gallager,” Low-Density Parity-check Codes”, 1963.

[15] AMIN SHOKROLLAHI, "LDPC Codes: An Introduction”, April 2, 2003.

\section{AUTHORS PROFILE}

Ms. Madhusmita Mishra : Received B.E Degree in Electronics and Communication Engineering from Utkal University, Orissa in 1997. She creditably completed her M.E Degree in Communication Control and Networking from R.G.P.V, Bhopal in 2005. She is serving the National Institute of TechnologyRourkela, India as a Research Scholar in the Department of Electronics and Communication Engineering from 2009 onwards. Her specialization is focused on Communication System Design.

Prof. Sarat Kumar Patra: Received Bsc (Engg.) from UCE Burla in Electronics and Telecommunication Engg. discipline. After completion of his graduation he served for India's prestigious Defense Research Development Organization (DRDO) as a scientist. He completed M.Tech at NIT Rourkela (Formerly known as REC Rourkela) in Communication Engg. Specialization in 1992. He received $\mathrm{PhD}$ from University of Edinburgh, UK in 1998. He has been associated with different professional bodies such as senior member of IEEE, Life Member IETE (India), IE (India), CSI (India) and ISTE (India). $\mathrm{He}$ has published more than 70 international journal and conference papers. Currently he is working as Professor in the Department of Electronics \& Communication Engineering at NIT Rourkela. His Current research area includes mobile and wireless communication, Communication Signal processing and soft computing.

Prof. Ashok Kumar Turuk:Dr Ashok Kumar Turuk received his $\mathrm{BE}$ and $\mathrm{ME}$ in Computer Science and Engineering from National Institute of Technology, Rourkela(Formerly Regional Engineering College, Rourkela) in the year 1992 and 2000 respectively. He obtained his $\mathrm{PhD}$ from IIT, Kharagpur in the year2005.Currently he is working as Associate Professor in the Department of Computer Science \& Engineering at NIT Rourkela. His research interest includes Ad-Hoc Network, Optical Network, Sensor Network, Distributed System and Grid Computing. 